\title{
Swallow syncope associated with complete atrioventricular block and vasovagal syncope
}

\author{
H Kakuchi, N Sato, Y Kawamura
}

\begin{abstract}
A 21 year old man presented with multiple, recurrent episodes of complete atrioventricular (AV) block associated with swallowing. Electrophysiological study revealed an AV block with swallowing of carbonated beverages and balloon inflation in the lower oesophagus. Evaluation did not demonstrate any underlying oesophageal or cardiac disease, and the AV block was not induced after intravenous atropine administration. The AV block was probably caused by a hypersensitive vagotonic reflex triggered by mechanical receptors in the lower oesophagus, resulting in suppression of the AV node. Head up tilt test revealed an increase in the high frequency spectrum of heart rate variability before the onset of the syncope. These findings suggest that the amplitude of the continual fluctuations in response to a variety of stimuli and derangement from both intrinsic and extrinsic environments was greater in this patient than in normal subjects. Swallow syncope is an unusual but treatable disorder. These reflexes that become exaggerated to the point of causing illness are poorly understood. (Heart 2000;83:702-704)
\end{abstract}

Keywords: swallow syncope; AV block; electrophysiological study; head up tilt test

Accepted 29 February 2000
A 21 year old man was referred to our hospital for evaluation of multiple, recurrent episodes of syncope associated with swallowing. He noted an augmentation and subsequent attenuation of symptoms during and after swallowing, which resolved as he stopped swallowing. These symptoms were always associated with swallowing large boluses of food or large amounts of beverages, especially carbonated beverages. He had no history of illness, and was not taking any medication. His heart rate was 67 beats per minute, and his blood pressure was $140 / 64 \mathrm{~mm} \mathrm{Hg}$. Cardiac examination, chest $x$ ray, echocardiography, oesophageal radiography, and ECG on admission were normal. Multiple episodes of symptomatic and asymptomatic paroxysmal atrioventricular (AV) block without escape beats were recorded repeatedly during meals or while drinking water on Holter ECG. He achieved a Bruce stage 5 during a treadmill exercise test and his heart rate reached 178 beats per minute without prolongation of the PQ interval. Sinus bradycardia was induced by carotid sinus massage, Valsalva manoeuvre, and gag reflex, but AV block was not induced by any of these.

Figure 1 shows complete AV block, of which the maximum RR interval was three seconds, and was induced by swallowing large amounts of carbonated beverages. Intravenous atropine at a dose of $2.5 \mathrm{mg}$ completely abolished the AV block, but the effect of isoprenaline was incomplete. On the other hand, $0.2 \mathrm{mg}$ of propranolol worsened the AV block. Oral atropine was discontinued due to the patient's complaint of thirstiness.

Figure 2 shows the results of the head up tilt test. Tilting to $80^{\circ}$ caused an initial increase in heart rate without a significant drop in blood pressure. Then, systolic blood pressure decreased slowly with rhythmic fluctuations, finally leading to syncope. Shortly before the onset of syncope, the heart rate gradually decreased. The spectral indices of heart rate variability (HRV) using fast Fourier analysis revealed that the ratio of the low frequency spectrum (LF) to the high frequency spectrum (HF) increased immediately after the head up tilt while the HF decreased. Finally, the HF increased and the $\mathrm{LF} / \mathrm{HF}$ decreased starting from just one minute before the onset of syncope. Oral disopyramide and intravenous atropine were not effective in inhibiting the syncope induced by the head up tilt test.
Figure 1 ECG during swallowing carbonated beverage. (A) Swallowing is followed by three seconds of complete AV block. (B) After the intravenous administration of $2 \mathrm{mg}$ of atropine, the AV block is completely blocked. 

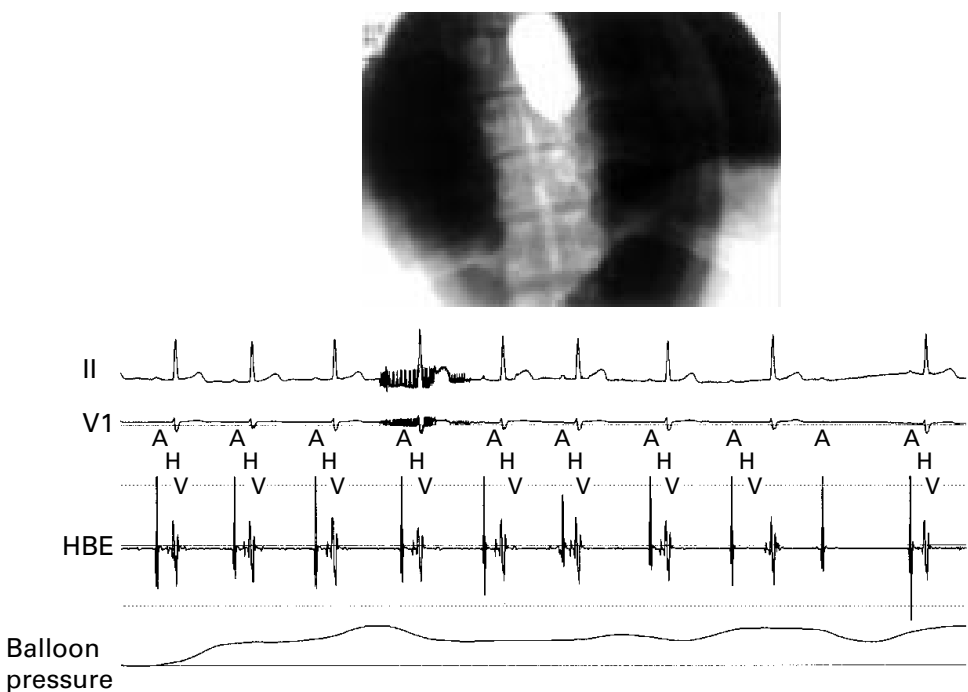

Figure 2 Intracardiac ECG during swallowing carbonated beverage. The AH intervals were prolonged transiently immediately after swallowing.

An electrophysiological study demonstrated normal $\mathrm{AH}$ and $\mathrm{HV}$ intervals $(75$ and $50 \mathrm{~ms}$, respectively). Incremental atrial pacing revealed the AV nodal decremental conduction property at a pacing rate of 150 beats per minute. Atrial extrastimulus testing revealed normal AV nodal function and effective refractory periods. Swallowing of carbonated beverages and inflation of a rubber balloon situated in the lower oesophagus to a varying degree of inflation volumes produced paroxysmal AV block (fig 3), which was shown to be an $\mathrm{AH}$ block by the His bundle ECG study. The cardiac catheterisation study, left ventriculography, and coronary angiography were normal.

\section{Discussion}

The neurally mediated syncopal syndromes encompass a number of apparently related dis-

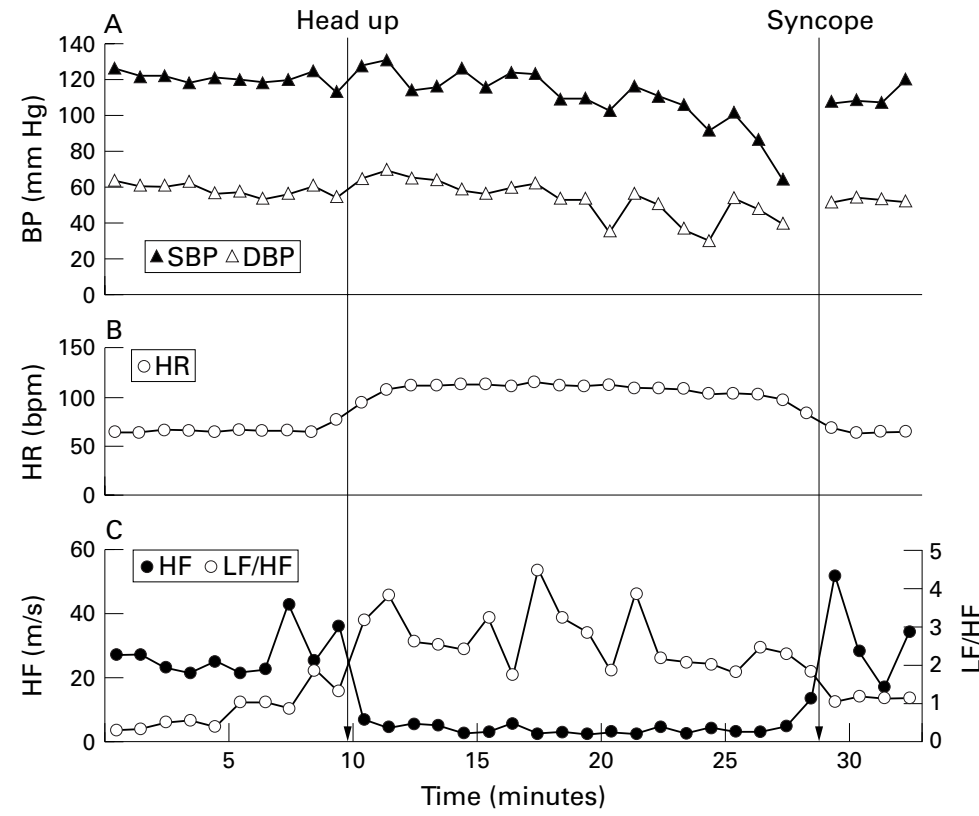

Figure 3 Sequential changes in heart rate (HR), systolic blood pressure (SBP), diastolic blood pressure (DBP), amplitude of high frequency ( $H F)$, and ratio of low frequency ( $L F$ ) amplitude to $\mathrm{HF}$ amplitude ( $\mathrm{LF} / \mathrm{HF})$. The spectral component at $0.25 \mathrm{~Hz}$ was defined as the HF component, whereas at 0.03 to $0.15 \mathrm{~Hz}$ it was defined as the LF component. turbances of reflex cardiovascular control characterised by transient inappropriate bradycardia and/or vasodilatation of the vascular bed. Although vasovagal syncope occurs very frequently, swallow or deglutition syncope is an unusual condition whereby temporary loss of consciousness occurs with swallowing.

We present a case of swallow syncope associated with complete AV block and vasovagal syncope. Although about 30 patients with swallow syncope have been reported in the literature, this is the first case that presented with both swallow syncope and vasovagal syncope.

It has been shown that most patients with swallow syncope have a variety of disorders, such as myocardial infarction, rheumatic carditis, digoxin toxicity, and gastro-oesophageal disorders (for example, hiatus hernia, gastric and oesophageal diverticulae, achalasia, spasm, and carcinoma of the oesophagus). A minority of patients are found to have no cause. ${ }^{12}$

Recent studies suggest that cardioinhibitory control neurons lie in an area between the nucleus ambiguus and the ventromedial aspect of the trigeminal spinal nucleus. ${ }^{6}$ Sensory fibres lie in fascicles in close proximity to each other as they pass the jugular ganglion and then disperse evenly within the nerve after passing the nodose ganglion. ${ }^{7}$ Vagal fibres innervate primarily the SA node, atrial musculature, and AV node, inhibiting AV conduction without any direct effect on the ventricular conduction system or myocardial contraction. ${ }^{8}$ Physiologically, swallow syncope is produced by a vagovagal reflex. With balloon inflation at the lower oesophagus, the afferent barrage is from distension of the oesophagus. Further from the results of the atropine administration, at least some of the necessary synapses in the arc were central and could be interrupted by atropine.

Head up tilt test suggests that this patient has a disturbance of the system described above. The normal compensatory response to head up posture is reflex tachycardia, more forceful contraction of the left ventricle, and vasoconstriction. However, in individuals with neurally mediated syncope, this forceful ventricular contraction in the setting of a relatively empty cavity may activate the cardiac mechanoreceptors. ${ }^{10}$ An afferent pathway consisting of unmyelinated left ventricular vagal $C$ fibres transmits signals to specific central nervous system sites. This reduces the efferent sympathetic tone and results in reflex hypotension and/or bradycardia. ${ }^{9}$ Hayano et al reported that the $\mathrm{HF}$ amplitude of the heart rate rapidly decayed with head up tilt and increased quickly with tilt back to the supine position. ${ }^{3}$ It has been shown that the LF amplitude of the blood pressure and heart rate shows rhythmic fluctuations at an interval of 48 to 100 seconds during head up tilt. This patient showed a rhythmic fluctuation in the LF amplitude of the heart rate. ${ }^{45}$ Furthermore, the $\mathrm{LF} / \mathrm{HF}$ ratio was greater and the $\mathrm{HF}$ amplitude was smaller than those in normal subjects immediately after head up tilt. These findings mean that the first amplitude of the continual fluctuations in response to a variety of stimuli and derangement from both intrinsic and extrinsic environments in this 
patient is greater than that in normal subjects. Thus, a disturbance in the autonomic feedback loop of the cardiovascular reflex led to the observed neurally mediated syncope and swallow syncope in this patient.

Swallow syncope has been successfully treated with anticholinergic agents, surgical denervation of portions of the oesophagus, and demand mode transvenous pacemaker placement. Planned treatment for our patient was permanent pacemaker implantation, with $\beta$ blockers.

1 Swallow syncope: report of a case and review of the literature. Neurology 1972;22:1086-93.

2 Eddy DP. The abnormal uppergastrointestinal vagovagal reflexes that affect the heart. Am $\mathcal{F}$ Gastroenterol 1976;66: 513-22.
3 Hayano J, Taylor JA, et al. Continuous assessment of hemodynamic control by complex demodulation of cardiovasdynamic control by complex demodulation of cardion
cular variability. Am f Physiol 1993; 264:H1229-38.

4 Murata K, Landriga PJ, Araki S. Effects of age, heart rate, gender, tobacco and alcohol ingestion on R-R interval variability in human ECG. F Auton Nerv Syst 1992;37:199206.

5 Hayano J, Sakakibara Y, Yamada A, et al. Accuracy of assessment of cardiac vagal tone by heart rate variability in normal subjects. Am f Cardiol 1991;67:199-204.

6 Gunn CG, Sevelius G, Puiggari MJ, et al. Vagal cardiomotor mechanisms in the hindbrain of the dog and cat. $A m \mathcal{F}$ Physiol 1968;214:258-62

7 Hurst JW, Logue RB. The heart, arteries, and veins. New York: McGraw-Hill Book Company, 1966.

8 Vassalle M, Caress DL, Slowin AJ, et al. On the cause of ventricular asystole during vagal stimulation. Circ Res 1967;20:228-41.

9 Toren PN. Role of cardiac vagal C-fibers in cardiovascular control. Rev Physiol Biochem Pharmacol 1979;86:1-94.

10 Thoren PN. Characteristics of left ventricular receptors with non-medullated vagal afferents in cats. Circ Res 1977;40: 415-21.

\section{IMAGES IN CARDIOLOGY}

\section{Giant coronary sinus and absent right superior vena cava}

This 66 year old woman underwent investigation for atypical chest pain. Chest radiography showed cardiomegaly, and echocardiography showed a giant coronary sinus $4 \mathrm{~cm}$ in diameter and larger than the aortic root and the left atrium, as shown on the parasternal long axis view (top). Intravenous injection of agitated saline from either the left or right brachial vein showed contrast enhancement of the giant coronary sinus. Venous angiography with contrast material injected simultaneously from both the right and left brachial veins showed that the right superior vena cava was absent and the contrast material entered the right atrium via a persistent left superior vena cava and the giant coronary sinus (bottom).

A persistent left superior vena cava is not an uncommon anomaly and usually drains into the right atrium through the coronary sinus which is dilated, measuring about 1 to $2 \mathrm{~cm}$ in diameter. In the rare setting of an absent right superior vena cava, this anomaly produces a truly giant coronary sinus because of the severely increased flow into the coronary sinus. Although this malformation can be associated with other cardiac anomalies, it may be an isolated finding as in this case and not associated with significant haemodynamic consequences. Recognition of this anomaly is important to avoid problems in venous catheterisation, pacemaker insertion, and cardiopulmonary bypass. (Ao, aorta; CS, coronary sinus; LA, left atrium; LV, left ventricle; $\mathrm{RA}$, right atrium.)
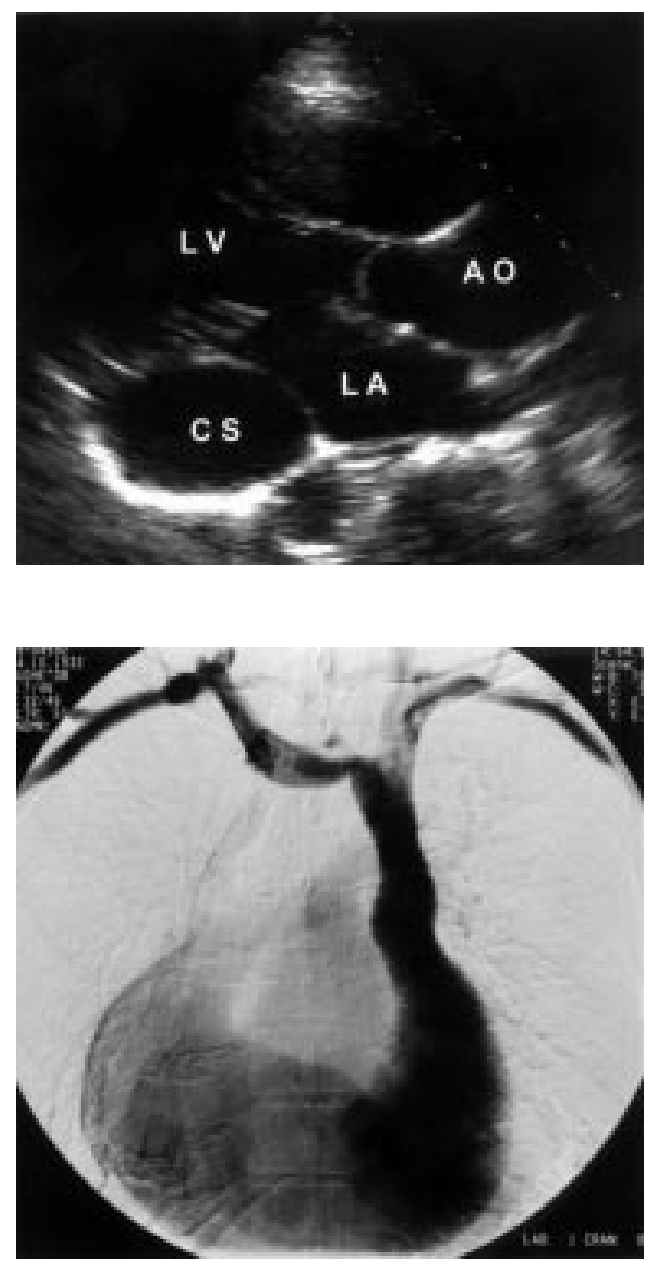